\title{
Real-Time Impedance Assay to Follow the Invasive Activities of Metastatic Cells in Culture
}

BioTechniques 33:842-850 (October 2002)

\author{
Charles R. Keese ${ }^{1,2}$, Kaumudi \\ Bhawe ${ }^{1}$, Joachim Wegener, \\ and Ivar Giaever ${ }^{1,2}$ \\ ${ }^{1}$ Rensselaer Polytechnic Insti- \\ tute, Troy, NY, ${ }^{2}$ Applied Bio- \\ Physics, Troy, NY, and ${ }^{3}$ Univer- \\ sity of Münster, Münster, \\ Germany
}

\begin{abstract}
Here we present research detecting the invasive activities of metastatic cells in vitro using electric cell-substrate impedance sensing $\left(E_{C I}{ }^{\mathrm{TM}}\right)$. The assay is based on previous microscopic observations, where metastatic cells added over established endothelial cell layers were observed to attach to and invade the cell layer. Human umbilical vein endothelial cells (HUVECs) were first grown to confluence on small gold electrodes. The impedance of these electrodes was followed after the addition of suspensions of different sublines of the Dunning murine prostatic adenocarcinoma series ( $G, A T 1, A T 2, A T 3$, $M L$, and $M L L)$. For highly metastatic sublines, within an hour after being challenged, the impedance of the confluent HUVEC layer was substantially reduced. The effect of the weakly metastatic sublines was less pronounced, and the extent and the rate of this drop in impedance could be correlated with the metastatic potential of each of six sublines tested. The real-time assay is effective in both normal and low (1\%) serum concentrations, and the detected activity requires the presence of viable transformed cells. In addition to the murine cell lines, similar behavior was observed using four established human prostatic cancer lines (DU145, PC3, TSU, and PPC1). These results suggest that this
\end{abstract}

ECIS-based assay might be used with primary human cultures to establish the metastatic abilities of cells isolated from biopsies.

\section{INTRODUCTION}

The growth of large solid tumors is dependent on an aberrant cell exhibiting uncontrolled proliferation and expressing the genes required to recruit endothelial cells to undergo angiogenesis, allowing the mass to grow beyond the constraints of passive diffusion (10). Notwithstanding, these behaviors alone do not result in multiple tumor formation - the hallmark of most malignant cancers. For secondary tumor formation via a blood-borne route, some tumor cells leave the primary mass, disrupt the basement membrane of capillary endothelial cells, affect the retraction of the endothelial cells, move through the intercellular junctions, and enter the circulatory system. After moving some distance from the primary tumor, these cell then attach to or become arrested near distant endothelial cells, traverse the spaces between these cells, disrupt the basement membrane structure, and migrate into the underlying tissue (4). Uncontrolled proliferation again ensues to form a new secondary tumor. As these activities are repeated, multiple tumors are established and, if unheeded, ultimately result in the death of the organism.

This complex in vivo metastatic behavior involves the regulation and expression of many genes involved in such diverse activities as endothelial cell binding (14), cell signaling resulting in endothelial retraction (11), the synthesis and secretion of proteolytic enzymes (1), and cell locomotion (3). These various activities can be isolated and individually observed in vitro. In addition, these components can be collectively studied using tissue culture methods similar to those introduced by Kramer and Nicolson (13). Here a cell monolayer of bovine endothelial cells was first established and then exposed to cell suspensions of a variety of both tumorigenic and nontumorigenic cells. Cell behavior was studied using scanning and transmission electron microscopy as well as phase-contrast timelapse microscopy. When endothelial cell monolayers were challenged with highly metastatic cell lines, such as the B16 melanoma cells, observations were recorded that showed the binding of the cells to the endothelial cell layer, the retraction of the endothelial cell junctions, and finally the penetration of the cells through the endothelial monolayer. This in vitro sequence of activities has been suggested to represent similar invasive activities that take place during the metastatic process in vivo. The assay provides striking images of the behavior of the metastatic cell but, by nature, is difficult to quantify and cannot provide information in real time regarding the dynamics of the process. Since its introduction, this type of assay has been modified and used extensively to monitor transendothelial migration of tumor cells in vitro. Although many of these approaches have involved qualitative microscopic observations, others have employed radio or fluorescent labeling of the tumor cells to yield quantitative measurements.

Giaever and Keese (6) first described 
the electric cell-substrate impedance sensing (ECIS ${ }^{\mathrm{TM}}$ ) (Applied BioPhysics, Troy, NY, USA) method in 1984. Since then, the method has been used to provide information regarding real-time changes in cell morphology, including cell substrate interactions, cell motility, and cell layer barrier function (7-9, $12,15,16,18,19)$. Based on the published observations of endothelial cell behavior in response to metastatic cells mentioned above, one would anticipate large ECIS impedance changes correlated with these activities. One would also anticipate changes in cell movements due to the displacement and replacement of a portion of the endothelial cells on the measuring electrode with the metastatic cells. The purpose of the research presented here was to determine the efficacy of such an ECIS-based assay. If successful, then an impedance assay of this sort should provide real-time, automated, and quantitative information regarding these in vitro events.

\section{MATERIALS AND METHODS}

\section{ECIS}

The ECIS Model 100 and an earlier prototype version of the instrument were used for this work. To study cell behavior with this instrument, cells are grown in culture wells containing goldfilm surface electrodes; normal culture medium serves as the electrolyte. An approximate constant current source applies an ac signal of $1 \mu \mathrm{A}$, usually at $4 \mathrm{kHz}$, between a small active electrode (250- $\mu \mathrm{m}$ diameter) and a large counter electrode to complete the circuit. Voltage is monitored with a lock-in amplifier providing amplitude and phase data. In addition to reporting impedance, these data are also converted to resistance or capacitance, treating the cellelectrode system as a simple resistor and capacitor in series. As the cells attach and spread upon the small electrode, they constrict the current, forcing it to flow beneath and between the cells, resulting in large increases in impedance. The microampere currents and the resulting voltage drop of a few millivolts have no measurable effect on the cells, and hence, the monitoring of cell behavior is noninvasive (5).

\section{Cell Culture Procedures}

The Johns Hopkins Brady Urological Institute supplied the Dunning sublines in frozen ampoules. These were thawed and grown in RPMI 1640 medium (Invitrogen, Carlsbad, CA, USA) supplemented with $2 \mathrm{mM}$ L-glutamine and containing $10 \%$ FBS with $250 \mathrm{nM}$ dexamethasone and $50 \mu \mathrm{g} / \mathrm{mL}$ gentamicin in a $5 \% \mathrm{CO}_{2}, 37^{\circ} \mathrm{C}$, high-humidity atmosphere. The behavior of the various lines had been previously studied (3). The specific Dunning lines used in this work included the highly metastatic ML, MLL, and AT3 sublines; the weakly metastatic AT1 and AT2 sublines; and the essentially non-metastatic G subline. Passaging was with trypsin/ EDTA, and frozen stocks were prepared using standard procedures with medium containing $10 \%$ DMSO and 20\% FBS. Human prostatic cell lines (DU145, PC3, TSU, and PPC1) were also obtained from Johns Hopkins and handled in an identical manner. For ECIS studies, cells were taken from slightly subconfluent cultures $48 \mathrm{~h}$ after passage, and a monodisperse cell suspension was prepared using standard tissue culture techniques with trypsin/EDTA. These suspensions were equilibrated at incubator conditions before addition to the electrode-containing wells.

Human umbilical vein endothelial cells (HUVECs) and their growth medium were purchased from VEC Technologies (Rensselaer, NY, USA). Medium 199 contained $80 \mu \mathrm{g} / \mathrm{mL}$ heparin, endothelial growth factor, $10 \%$ FBS, and $50 \mu \mathrm{g} / \mathrm{mL}$ gentamicin. Passaging was with trypsin/EDTA and frozen stocks were prepared as described above. Endothelial cells were generally passaged 6-8 times, and then the stock of cells was replaced.

\section{Preparation and Initial Inoculation of Electrodes with HUVEC Suspensions}

Electrode arrays were supplied by Applied BioPhysics, where the goldfilm electrodes were fabricated using photolithographic techniques on Lexan polycarbonate supports. These were connected to the electronics as previously described (19). The completed arrays consisted of eight individual small electrodes $(250-\mu \mathrm{m}$ diameter) with larger counter electrodes located at the base of small wells (approximately $600 \mu \mathrm{L}$ volume with $0.8 \mathrm{~cm}^{2}$ substrate area) for cell culture. Each well was normally used with 400 or $450 \mu \mathrm{L}$ medium.

For most experiments, gelatin was used to precoat the ECIS wells; a solution of $200 \mu \mathrm{g} / \mathrm{mL}$ gelatin in $0.15 \mathrm{M}$ $\mathrm{NaCl}$ was used to flood the bottom of each well. After $15 \mathrm{~min}$ incubation to allow the gelatin to adsorb, the protein solutions were aspirated, and the electrode-containing wells were rinsed twice. They were then partially filled with $200 \mu \mathrm{L}$ HUVEC medium and allowed to equilibrate in the tissue culture incubator for 15-60 min.

A HUVEC suspension was prepared at $5 \times 10^{5}$ cells $/ \mathrm{mL}$, and $200 \mu \mathrm{L}$ were added to each well, resulting in a final surface concentration of $1.25 \times 10^{5}$ cells $/ \mathrm{cm}^{2}$ and a well volume of $400 \mu \mathrm{L}$.

Following inoculation of cells into ECIS wells, the attachment and spreading were followed by impedance measurements. The HUVECs were generally incubated for 1-2 days before the prostatic cell challenge. Impedance measurements were used to verify that confluence was achieved and maintained and that the cells exhibited normal levels of impedance fluctuations-indicative of healthy cell layers (15).

\section{Challenge of Endothelial Layers}

The Dunning cells were cultured to near confluence with regular feedings of RPMI medium. Monodisperse cell suspensions were prepared in fresh Medium 199 (HUVEC medium) at 20 $\times 10^{5}$ cells $/ \mathrm{mL}$, and $50 \mu \mathrm{L}$ were added to wells containing endothelial cell monolayers $(450 \mu \mathrm{L}$ total well volume). This resulted in a final surface concentration of Dunning cells of 1.25 $\times 10^{5}$ cells $/ \mathrm{cm}^{2}$ (one Dunning cell for each endothelial cell). The impedance of the challenged endothelial cell layer was monitored via ECIS for the next 12-20 h. Sometimes, following the measurement, the medium was aspirated and the cells were fixed with $10 \%$ formalin and stained with methylene blue. Identical procedures were used for the human lines. 


\section{Conditioned Dunning Medium}

Medium used to maintain confluent cultures of Dunning cells for $72 \mathrm{~h}$ was removed from culture vessels and centrifuged at $3000 \times \mathrm{g}$ for $10 \mathrm{~min}$ to remove any suspended cells. This medium was then used to replace $250 \mu \mathrm{L}$ medium removed from an ECIS well with a confluent HUVEC layer in place.

\section{RESULTS AND DISCUSSION}

Figure 1 shows the initial attachment of the HUVECs measured via time-course impedance changes. Seven individual electrodes are followed from the time of inoculation (time zero) to 20 $\mathrm{h}$ after inoculation. The initial rise in the curve is due to cell attachment and spreading that, for this lot of HUVECs, increased the resistive portion of the impedance at $4 \mathrm{kHz}$ from six to seven times that of the cell-free electrode. Spreading is completed in approximately $2.5 \mathrm{~h}$. The resistance fluctuations result from movement or undulations of the established cell sheet that constrain the current. These changes are both due to fluctuations in the transcellular barrier function and in the spacing between the basal side of the cell and the electrode surface (8). A second endothelial cell attachment curve using a different lot of HUVECs is shown in Figure 2 for four inoculated electrodes and also one control electrode that receives no cells. In these data, there is a difference in the behav- ior of the HUVECs on electrodes precoated with adsorbed gelatin compared to those where the adsorbed protein layer is simply a collection of those proteins found in the FBS used to supplement the growth medium. This response to gelatin-coated substrates was seen only for some specific lots of HUVEC tested. Nevertheless, as a tight layer (high impedance) was deemed best considering the nature of the experiments, in most work reported, the electrodes were precoated with gelatin.

Figure 3 (upper panel) is the first of several figures showing changes in the resistive portion of the impedance during the challenge of the endothelial cell layer, in this case with the highly metastatic Dunning MLL cells. The experimental curve is run in duplicate with complete medium containing 10\% FBS as described in the experimental methods. Data acquisition began at time zero (not shown) when the HUVEC layer was established and completely confluent. Impedance data recording is briefly paused at the first vertical mark on the horizontal axis. MLL cells $(50 \mu \mathrm{L}$ suspension) are then added, and at the second vertical mark (around 7.2 h) data acquisition resumes. Time measurement continues during this pause, and the resistance recorded at the start and end of the pause are connected with a straight line. The control curve received a sham at this time and is essentially unaffected by the addition of $50 \mu \mathrm{L}$ fresh Medium 199 without cells.

There is a substantial drop in the re-

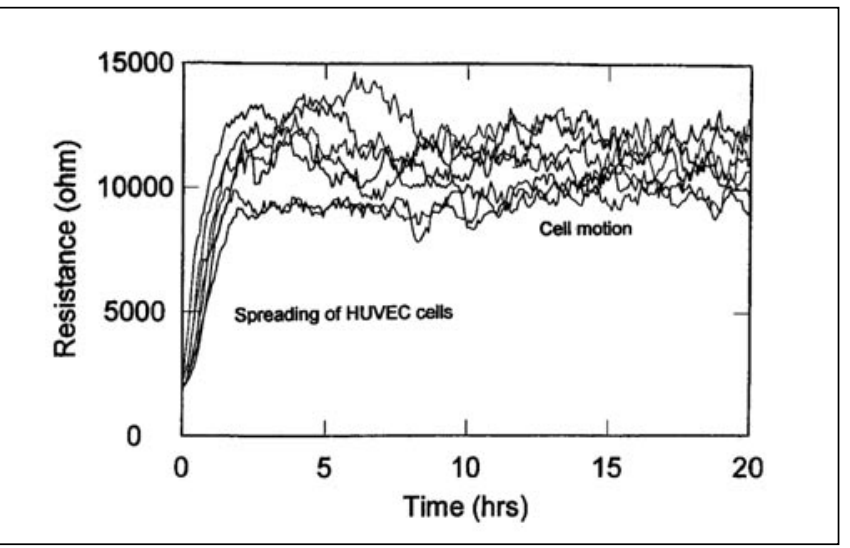

Figure 1. ECIS measurements of HUVEC inoculations. Measurements made in seven individual wells were at $4 \mathrm{kHz}$, and the resistive portion of the impedance is presented. All electrodes were precoated with gelatin. sistance of the wells exposed to the MLL cells over an approximately 2-h period. These changes are presumably due to direct interactions of the metastatic cells and endothelial monolayer, resulting first in retraction of the endothelial cell junctions, followed by extravasation of the metastatic cells to the substratum. After this drop, the sustained reduced resistance is that of the collection of HUVEC and MLL cells on the substratum. Microscopic examination of fixed and stained preparations of the cells a few hours after the challenge revealed a disorderly layer with some open areas and a considerable number of partially spread and rounded cells.

We were curious whether the withdrawal of serum from the medium would alter the systems response, as serum components might play a role in either promoting the metastatic cell activities or in stabilizing the endothelial cell layer. In the lower panel of Figure 3 , an experiment shows the ability of the assay to function at reduced serum concentration. In this experiment, HUVECs were inoculated and grown for two days in standard medium containing 10\% FBS. The confluent layer was then allowed to equilibrate in medium with only $1 \%$ FBS for several hours before the addition of the sham or the MLL cells in Medium 199 now with $1 \%$ FBS. As can be seen upon comparing these two figures, the lower serum concentration has resulted in a notice-

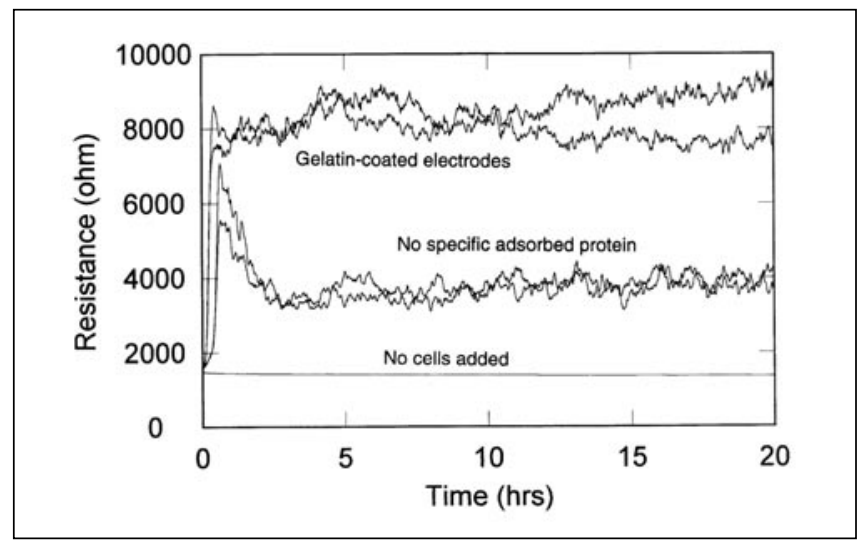

Figure 2. Effect of adsorbed gelatin coatings on HUVEC behavior. Measurements of the resistive portion of the impedance at $4 \mathrm{kHz}$ are shown following inoculation at time zero. Duplicate inoculations were made in wells having electrodes without special pretreatment and in wells where electrodes were precoated with adsorbed gelatin. This striking influence of substrate coating was observed for some lots of HUVECs; however, many lots showed a much less pronounced effect. The lower trace is of a well not receiving cells. 
able drop in the intact HUVEC layer resistance, from around $12000 \Omega$ at $10 \%$ FBS to about $7000 \Omega$ at the $1 \%$ level. Nevertheless, there is a substantial further drop elicited by the MLL cell suspension addition, as well as a quieting of the typical endothelial cell impedance fluctuations. Again, the sham has little effect on the control curve. The ability to work at low serum concentration or in defined medium could also prove useful in reducing or eliminating the interference of serum components in future mechanistic studies.

Figure 4 shows varying effects of different sublines of the Dunning series using this challenge assay. These data are normalized by dividing the resistive portion of the impedance by its value at time zero for each well (approximately $10000 \Omega$ ), providing an easy comparison of the fractional change in resistance. In the upper and lower panels of Figure 4, HUVEC layers are challenged with Dunning cells added in $50 \mu \mathrm{L}$ complete Medium 199 containing $20 \times 10^{5}$ cells $/ \mathrm{mL}$. The vertical lines are the same as in Figure 3, and a sham is added to the control curve. In the upper panel of Figure 4 , duplicate wells received the weakly metastatic $G$ subline and the highly metastatic AT3 subline. The G cell addition, unlike the previous MLL additions, brings about a smaller initial drop in resistance, and the resistance of the collection of cells undergoes partial reversal in this drop and displays some return of cell-induced impedance fluctuations. The AT3 challenge, although resulting in a less steep drop in impedance than MLL, results in curves eventually reaching resistance levels near that of cell-free electrodes and with nearly complete elimination of detectable cell motion (resistance fluctuations). The ML highly metastatic subline had behavior similar to that of MLL (data not shown). In the lower panel of Figure 4, three different Dunning strains are used, namely G, AT1, and AT2. In this run, the two electrodes with AT2 cells elicit a slightly more rapid drop in the resistance than do the electrodes with AT1 cells, although both sub-

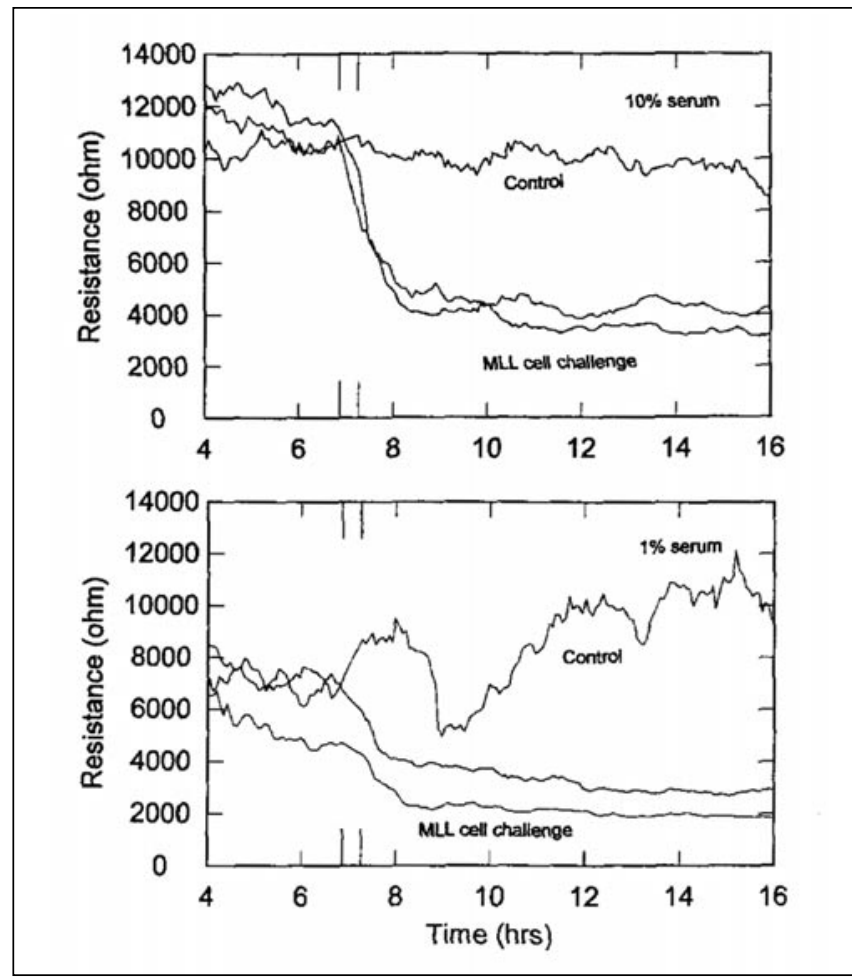

Figure 3. Resistance changes during MLL cell challenges. Resistance changes in the impedance at $4 \mathrm{kHz}$ as confluent layers of HUVEC cells are challenged with MLL Dunning cell suspensions in the presence of $10 \%$ and $1 \%$ FBS in the media. The control curve received medium without cells. lines reportedly have similar metastatic potential in vivo. With the exception of the lowest AT2 trace, these show impedance changes that are intermediate between the $G$ subline and the highly metastatic lines. The $\mathrm{G}$ cells again show the rebound pointed out in the upper panel of Figure 4.

Metalloproteinases and other soluble factors are known to play a role in the metastatic process. Work with murine sarcoma cells (20) and human pancreatic cancer cells (17) had shown that medium conditioned by the cancer cell lines induced endothelial cell retraction. Experiments were carried out to determine if the possible addition of soluble factors released by the metastatic cells would have an effect on the impedance of the HUVEC layer similar to that observed with the cell suspensions. These effects could not be observed under the conditions of our studies. Figure 5

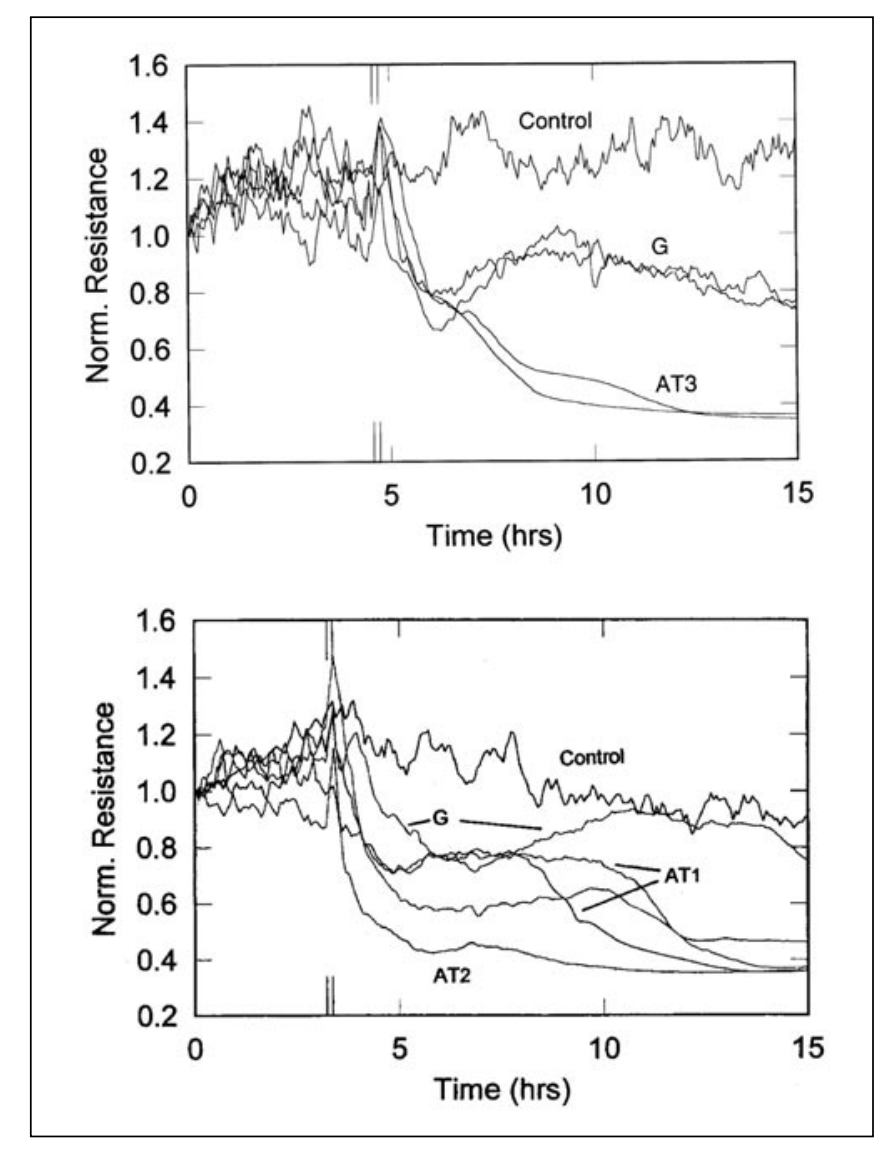

Figure 4. Normalized resistance changes during Dunning subline challenges. Normalized resistance changes in the impedance at $4 \mathrm{kHz}$ as confluent layers of HUVECs are challenged with suspensions of (upper panel) the $G$ and AT3 Dunning sublines and (lower panel) the G, AT1, and AT2 Dunning sublines (resistance is normalized to its starting value, approximately $10000 \Omega$ ). 
shows the ECIS response of HUVECs exposed to conditioned medium as well as whole cells. MLL cells added in 50 $\mu \mathrm{L}$ fresh medium bring about the clear drop in resistance. The control curve in this case had $250 \mu \mathrm{L}$ medium replaced with fresh medium (total volume 400 $\mu \mathrm{L}$ ), whereas the other trace had $250 \mu \mathrm{L}$ medium replaced with medium that was conditioned for three days by highly confluent MLL cultures.

Since large numbers of cells are being added during the challenge, we investigated the possibility that a reaction to the crowded conditions or contact with the cell membranes simply caused the HUVEC cells layer to be altered. To accomplish this, MLL cells were heat killed by exposure to $55^{\circ} \mathrm{C}$ for $15 \mathrm{~min}$ before addition to the established HUVEC culture. The results of this experiment are shown in Figure 6, where four traces are shown where established endothelial layers are exposed to cell suspensions or a sham at time zero and the normalized resistance is plotted against time. Here we see the normal response of the HUVECs to healthy MLL and the $G$ cell sublines, but the medium sham (control) and the heat-killed cell additions elicit no response and are indistinguishable in Figure 6. The fifth straightline trace is the normalized resistance measured from a well without cells. The slight drift in this curve is due to evaporation from the well and the resulting decrease in the resistivity of the medium.

Using a published model of the cellelectrode interaction (8), we have further analyzed the metastatic response to examine the behavioral changes taking place. The model was developed to calculate the specific impedance of a cellcovered electrode $\left(Z_{c}\right)$ as a function of the applied ac frequency (f). In the model, cells are treated as circular disks of radius $r_{c}$ and hovering a distance, $h$, over the electrode surface and bathed in medium of resistivity $\rho$. The specific impedance of the cell-free electrode $\left(Z_{n}\right)$ is assumed to remain constant and is experimentally measured. $Z_{\mathrm{m}}$ is the impedance to ac current flow directly through the two plasma membranes due to membrane capacitance, $\mathrm{C}_{\mathrm{m}}$. In the model the extracellular current flows radially in the spaces formed between the ventral surface of the cell and the electrode surface before exiting to solution through transcellular spaces.

Solution of the equations associated with the model gives the specific impedance of the cell-covered electrode as:

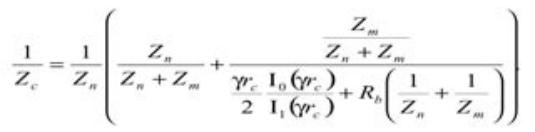

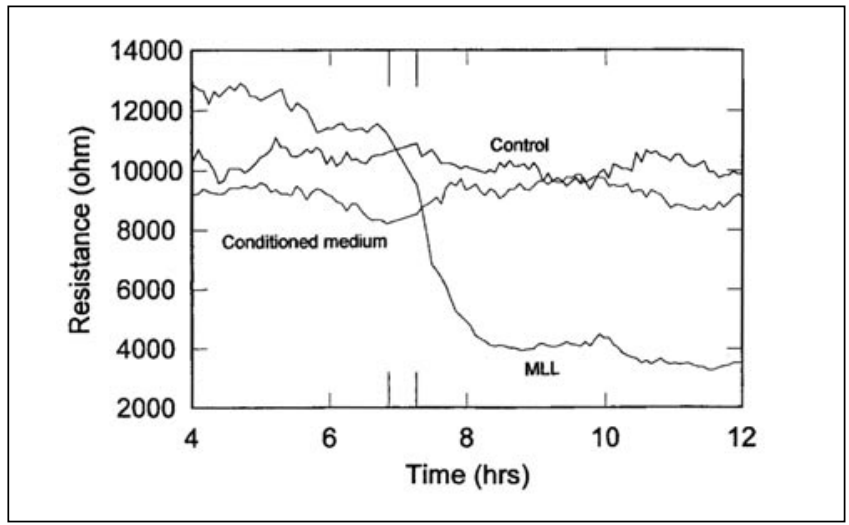

Figure 5. Resistance changes during MLL cell or conditioned medium challenges. Resistance changes in the impedance at $4 \mathrm{kHz}$, as confluent layers of HUVECs are challenged with MLL cell suspension and MLL-conditioned medium.

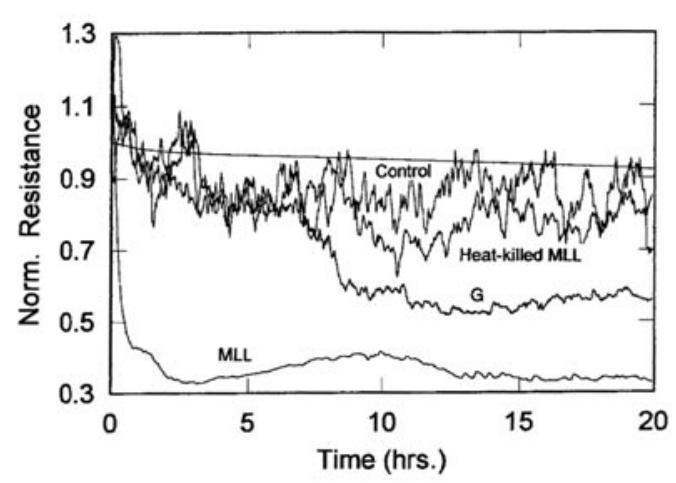

Figure 6. Normalized resistance changes during live or dead cell challenges. Challenge of HUVEC layers with both live and heat-killed MLL cells and with live $\mathrm{G}$ cells. The resistive portion of the impedance at $4 \mathrm{kHz}$ is normalized to its starting value (approximately $2000 \Omega$ without cells and $12000 \Omega$ for the four traces of confluent HUVEC layers).

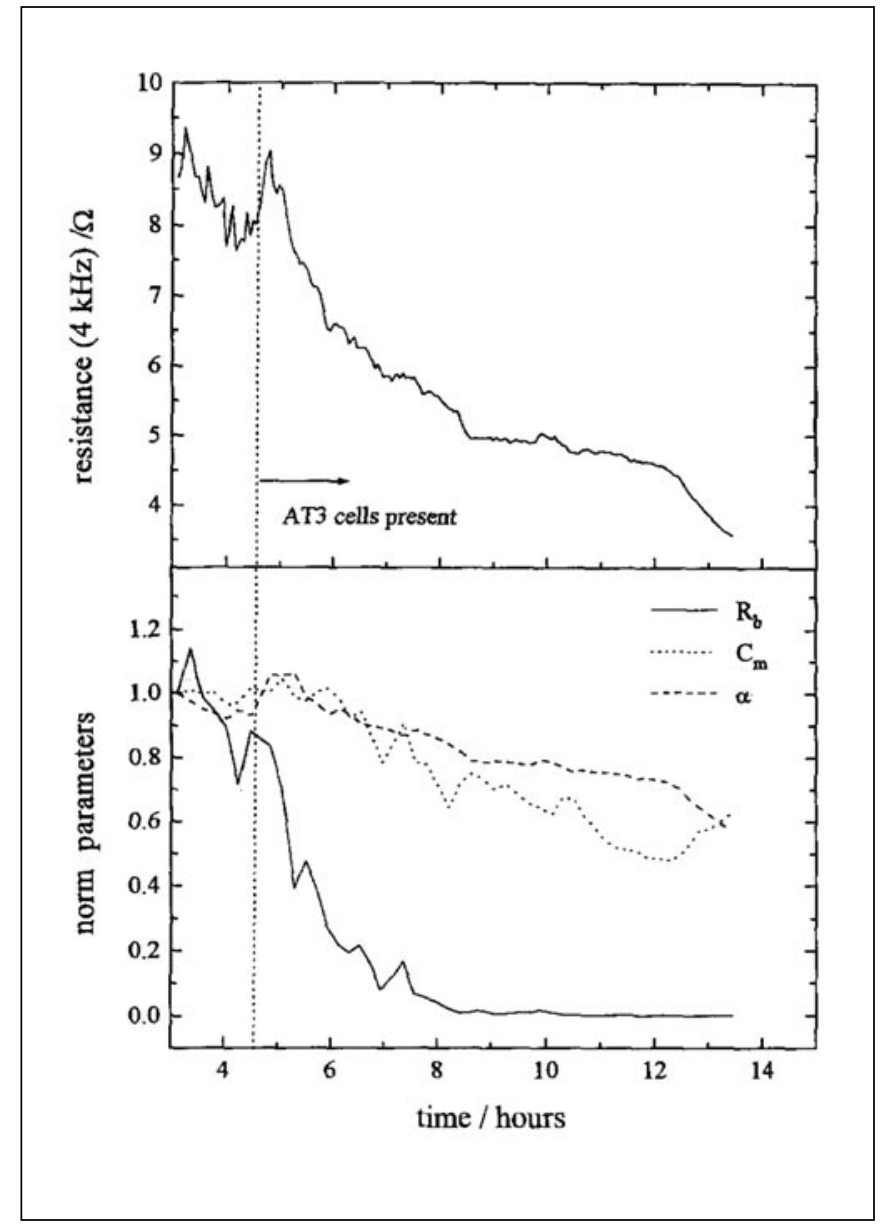

Figure 7. Changes in model parameters during AT3 cell challenge. A challenge of a confluent HUVEC layer showing the time course of resistance changes and changes in parameters based on a model of cell-induced impedance changes. 
where $\mathrm{I}_{0}$ and $\mathrm{I}_{1}$ are modified Bessel functions of the first kind of order 0 and 1 .

This solution depends on three parameters:

$\mathrm{R}_{\mathrm{b}}$, the resistance between the cells

for a unit area

$\alpha$, defined by:

$$
\gamma r_{c}=r_{c} \sqrt{\frac{p}{h}\left(\frac{1}{Z_{n}}+\frac{1}{Z_{m}}\right)}=\alpha \sqrt{\frac{1}{Z_{n}}+\frac{1}{Z_{m}}} .
$$

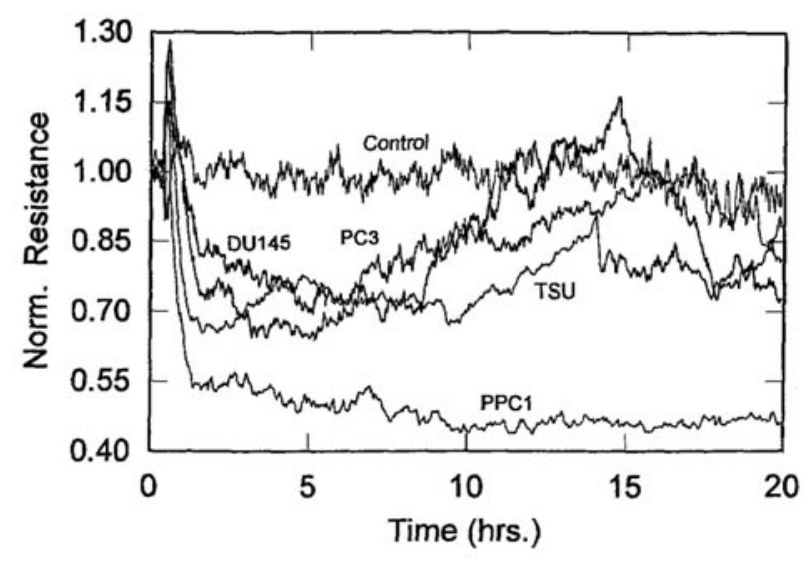

Figure 8. Prostatic cell challenge. HUVECs were challenged with several different human prostatic cell suspensions. The resistive portion of the impedance normalized to its value at time zero is presented.
$\mathrm{C}_{\mathrm{m}}$, where $\mathrm{C}_{\mathrm{m}}=-i /\left(\pi \mathrm{f} \mathrm{Z}_{\mathrm{m}}\right)$

Time-course data of a HUVEC-AT3 challenge were measured at three different ac frequencies (400, 4000, and $40000 \mathrm{~Hz}$ ). The time points were then fitted using the model. Reasonable values of the three parameters are first chosen and then used to calculate values of $\mathrm{Z}_{\mathrm{c}}$ and the corresponding values of the series resistance and capacitance at the three measuring frequencies. The calculated and experimental values are then compared and the model parameters altered to improve the fit. This is continued in an iterative process until an acceptable fit is obtained. These parameters are then used as starting values for analysis of the second time point in the series and so on. Figure 7 shows the raw data in the upper panel and the calculated changes in the three model parameters in the lower panel. Notice that most of the change associated with the metastatic cell challenge is in the para- 
meter $R_{b}$, the barrier function of the layer. This is presumably due to the retraction of the HUVECs from one another, opening up intercellular spaces for less obstructed current flow.

Some of the basic challenge experiments were also performed using a base layer of MDCK epithelial cells (data not shown). Although this base layer usually produced results similar to those described above with HUVECs, considerable variation was found from experiment to experiment. We believe this variation can be attributed to the very high barrier function of MDCK monolayers that can be an order of magnitude higher than that of the endothelial cells. A similar size retraction of the cell intercellular spaces will result in a considerably larger percentage drop in impedance for the MDCK than for the HUVEC layer. This very high sensitivity to the challenge could reflect subtle variations in experimental conditions not seen with the HUVEC layers. Another epithelial cell line, BSC-1, essentially did not respond to the metastatic cell challenge. This lack of response could be biological in nature - a failure of cell-cell interactions to result in the behavior observed with HUVECs. It is also possible that the weaker barrier function of the BSC-1 cells diminishes the ECIS response to the retraction of the intercellular spaces as described above. Although HUVEC cultures are more demanding in their culture requirements, these monolayers both responded strongly to the metastatic cells and gave results that were consistent from experiment to experiment.

We have carried out challenges with several human prostatic cancer cell lines. Results of confluent HUVEC monolayers challenged with $10^{5}$ cells $/ \mathrm{cm}^{2}$ DU154, PC3, PPC1, and TSU lines are shown in Figure 8. The PPC1 line clearly results in the most rapid and complete disruption of the endothelial cell layer. The other lines seem fairly similar, but one can discern possible differences in the slope and size of the initial drop, the degree of fluctuations in the signals, and the final impedance level obtained. We hope in the future to determine if these more subtle observations are reproducible and if they can be related to the in vivo behavior of the lines.

The ECIS instrumentation is now being adapted to monitor the activities of endothelial cells under both laminar and turbulent flow (2). It will be of interest to see if the behaviors described above are altered under these conditions encountered during the metastatic process in vivo.

\section{ACKNOWLEDGMENTS}

This work was performed in part pursuant to a contract with the National Foundation for Cancer Research. Support was also in the form of an SBIR Phase I and II Grant from the National Cancer Institute. We are thankful for the excellent technical assistance of Narayan Karra and Lavanya Reddy and thank Professor Donald S. Coffey of Johns Hopkins for useful technical discussions.

\section{REFERENCES}

1.Brooks, P.C., S. Stromblad, L.C. Sanders, T.L. vonSchalscha, R.T. Aimes, W.G. Stetler-Stevenson, J.P. Quigley, and D.A. Cheresh. 1996. Localization of matrix metalloproteinase MMP-2 to the surface of invasive cells by interaction with integrin alpha $\mathrm{v}$ beta 3. Cell 85:683-693.

2.DePaola, N., J.E. Phelps, L. Florez, C.R. Keese, F.L. Minnear, I. Giaever, and P.I. Vincent. 2001. Electrical impedance of cultured endothelium under fluid glow. Ann. Biomed. Eng. 29:1-9.

3.Epner, D.E., A.W. Partin, J.A. Schalkan, J.T. Isaacs, and D.S. Coffey. 1993. Association of glyceraldehyde-3-phosphate dehydrogenase expression with cell motility and metastatic potential of rat prostatic adenocarcinoma. Cancer Res. 53:1995-1997.

4.Fidler, I.J. and R. Radinsky. 1990. Genetic control of cancer metastasis. J. Natl. Cancer Inst. 82:166-168.

5.Ghosh, P.M., C.R. Keese, and I. Giaever. 1994. Morphological response of mammalian cells to pulsed ac fields. Bioelectrochem. Bioenergetics 33:121-133.

6.Giaever, I. and C.R. Keese. 1984. Monitoring fibroblast behavior with an applied electric field. Proc. Natl. Acad. Sci. USA 81:37613764.

7.Giaever, I. and C.R. Keese. 1986. Use of electric fields to monitor the dynamical aspect of cell behavior in tissue culture. IEEE Trans. Biomed. Eng. 33:242-247.

8.Giaever, I. and C.R. Keese. 1991. Micromotion of mammalian cells measured electrically. Proc. Natl. Acad. Sci. USA 88:7896-7900. (Erratum 90:1634.)

9.Giaever, I. and C.R. Keese. 1993. A morphological biosensor for mammalian cells. Nature 366:591-592.

10.Hahnfeldt, P., D. Panigrahy, J. Folkman, and L. Hlatky. 1991. Tumor development un- der angiogenic signaling: a dynamical theory of tumor growth, post-vascular dormancy and treatment response. Cancer Res. 59:47704775

11.Honn, K.V., D.G. Tang, I. Grossi, Z.M. Duniec, J. Timar, C. Renaud, M. Leithauser, I. Blair, et al. 1994. Tumor cellderived 12(S)-hydroxyeicosatetraenoic acid induces microvascular endothelial-cell retraction. Cancer Res. 54:565-574.

12.Keese, C.R., N. Karra, B. Dillon, A. Goldberg, and I. Giaever. 1998. Cell-substratum interactions as a predictor of vytotoxicity. In Vitro Toxicol. 11:83-192.

13.Kramer, R.H. and G.L. Nicolson. 1979. Interactions of tumor cells with vascular endothelial cell monolayers: a model for metastatic invasion. Proc. Natl. Acad. Sci. USA 76:5704-5708.

14.Lafrenie, R.M., T.J. Podoe, M.R Buchanan, and F.W. Orr. 1992. Up-regulated biosynthesis and expression of endothelialcell vitronectin receptor enhances cancer celladhesion. Cancer Res. 52:2202-2208.

15.Lo, C.M., C.R. Keese, and I. Giaever. 1993. Monitoring motion of confluent cells in tissue culture. Exp. Cell Res. 204:102-109.

16.Mitra, P., C.R. Keese, and I. Giaever. 1991. Electric measurements can be used to monitor the attachment and spreading of cells in tissue culture. BioTechniques 11:504-511.

17.Okamoto, H., S. Nakamori, M. Mukai, K. Shinkai, H. Ohigashi, O. Ishikawa, H. Furukawa, S. Imaoka, et al. 1998. Down-regulation of focal adhesion kinase, pp125 (FAK), in endothelial cell retraction during tumor cell invasion. Clin. Exp. Metastasis 16:243-252.

18.Tiruppathi, C., A.B. Malik, P.J. Del Vecchio, C.R. Keese, and I. Giaever. 1992. Electrical method for detection of endothelial cell shape change in real time. Proc. Natl. Acad. Sci. USA 89:7919-7923.

19.Wegener, J., C.R. Keese, and I. Giaever. 2000. Electric cell-substrate impedance sensing (ECIS) as a noninvasive means to monitor the kinetics of cell spreading to artificial surfaces. Exp. Cell Res. 259:158-166.

20.Yasuda, T., H. Matsui, M. Kanamori, K. Yudoh, K. Ohmori, M. Aoki, and H. Tsuji. 1999. Effects of tumor cell-derived interleukin 1 alpha on invasiveness of metastatic clones of murine RCT sarcoma through endothelial cells. Tumor Biol. 20:105-116.

Received 6 February 2002; accepted 16 July 2002.

\footnotetext{
Address correspondence to:

Dr. Charles R. Keese

Department of Biology

Rensselaer Polytechnic Institute

Troy, NY 12180, USA

e-mail:keesec@rpi.edu
}

For reprints of this or any other article, contact Reprints@BioTechniques.com 